Burkitt's lymphoma

\title{
Clues to the role of malaria
}

\section{from M.A. Epstein}

STEADILY accumulating evidence that Epstein-Barr (EB) virus plays a crucial role in the chain of events which causes Burkitt's lymphoma in endemic zones culminated in the compelling findings of the World Heath Organization's prospective study in Uganda ${ }^{1}$. The virus seems to play its part by infecting and thereby 'immortalizing' a pool of B lymphocytes. Their continuous cell divisions facilitate any one of three characteristic chromosomal translocations that can activate the c-myc oncogene ${ }^{2}$. However, since EB virus is ubiquitous whereas Burkitt's lymphoma has a high incidence only in remarkably restricted areas, it has long been recognized that a co-factor must determine the geographical distribution of the endemic tumours. Already fifteen years ago Denis Burkitt suggested that hyperendemic malaria was the co-factor and collected much supporting epidemiological evidence $^{3}$. But possible mechanisms for its mode of action have remained obscure. On page 449 of this issue Whittle et al. ${ }^{4}$ provide the first clues.

Like all other herpes viruses, EB virus gives rise to a life-long infection to which the healthy carrier develops specific immunological responses. These are excellent at damping down the virus but never enough to eliminate it from the, as yet unidentified, immunologically-privileged site in which it persists. Thus, a delicate balance evolves. On the host side, the balance depends both on virus-neutralizing antibodies, which restrict the spread of the agent from one target cell to another, and on a full cellular immunological response, which is capable of destroying infected cells because they display viral antigens.

During primary EB virus infection, natural killer cells and other non-specific $T$ cells may be of immediate importance. But thereafter, specific cytotoxic $\mathrm{T}$ lymphocytes (which are HLA-restricted) play a central role in maintaining life-long surveillance of the viral carrier state ${ }^{5}$. The very great importance of this surveillance is shown by the dire consequences when it fails: genetic defects lead to death from uncontrolled polyclonal proliferation of EB virus-immortalized cells, and widespread virus replication follows primary infection. Therapeutic immunosuppression in organ graft recipients also carries a considerable risk of malignant lymphoma ${ }^{6}$.

Although it has been known in general terms that malaria has profound effects on immune reactivity ${ }^{7}$, Whittle et al. ${ }^{4}$ now clearly demonstrate that the control of EB virus-specific $T$ lymphocytes is dramatically impaired during acute malarial infection by Plasmodium falciparum. Interestingly, this impairment resembles that of immunosuppressed renal allograft patients ${ }^{8}$. Moreover, while looking for changes in lymphocyte numbers that might explain the phenomenon, Whittle and colleagues have recorded further intriguing observations. Not only are $\mathrm{T}$ cell numbers decreased and B cells numbers increased, as might be expected, but the percentage of $\mathrm{T}$-helper (T4) cells is reduced in relation to $\mathrm{T}$-suppressor (T8) cells, giving a low T4/T8 ratio. It is not clear how malaria brings about these changes but the loss of T4 cells is not due to cytotoxic antibodies present in the acute phase serum.

A reduced T4/T8 ratio is one of the key hallmarks of acquired immune deficiency syndrome (AIDS), in which T4 lymphocytes are infected and destroyed by the retrovirus known as LAV $^{9}$ or HTLV III ${ }^{10}$. Whittle et al. make the interesting speculation that a latent retrovirus of this group, after activation by malaria, might similarly account for the depressed $\mathrm{T} 4 / \mathrm{T} 8$ ratios in their malaria patients.

However the damage to specific T-lymphocyte control in malaria arises, it is not difficult to envisage that in the hyperendemic disease, with its repeated attacks throughout each and every year, such damage will favour the unrestrained growth of EB virus-carrying B cells in a substantial number of individuals. A firm immunological explanation for the geographical coincidence of endemic Burkitt's lymphoma with hyperendemic malaria has thus been provided by experiments of Whittle et al., even though the exact cause has not yet been identified.

Other loose ends remain to be tied up. Even where EB virus and hyperendemic malaria coexist in populations, the results of the prospective study in Uganda suggest that certain individuals are at special risk. Evidence for a genetic predisposition has not been forthcoming and should perhaps be more rigorously sought for. It has also been suggested that infection by the virus very early in life might be a predisposing factor ${ }^{11}$; this too requires clarification. Alternatively, some special sequential relationship between the primary viral infection and the malarial infection could be important either in determining damage to the EB virus-specific T cells, or in providing unusual target $\mathrm{B}$ cells, which more readily escape from the damaged control mechanisms.

1. De-Thé, G. et al. Nature 274, 756 (1978).

Klein, G. Cell 32, 311 (1983)

Burkitt, D.P. J. natl. Cancer Inst. 42, 19 (1969)

4. Whittle, H.C., Brown, J., Marsh, K., Greenwood, B.M. Seidelin, P., Tighe, H. \& Wedderburn, L. Nature 312, 449 (1984).

5. Rickinson, A.B. et al. Cancer Res. 41, 4216 (1982)

6. Klein, G. \& Purtilo, D. T. (eds) Cancer Res. 41 Suppl., 4209 (1981).

. Weidanz, W.P. Br. med. Bull. 38, 167 (1982)

8. Gaston, J.S.H., Rickinson, A.B.\& Epstein, M. A. Lancet i, 923-935 (1982)

9. Barré-Sinoussi, F. et al. Science 220, 868 (1983)

10. Gallo, R.C. et al. Science 224, 500 (1984).

11. De-Thé, G. Lancet i, 335 (1977)

M.A. Epstein is at the University of Bristol Medical School, University Walk, Bristol BS8 ITD, UK.

\section{Mathematics}

\section{Five bodies to infinity}

\section{from Ian Stewart}

TYCHO Brahe's observations of planetary motion led Johannes Kepler to the idea that planets orbit the Sun in ellipses. Isaac Newton showed that this would follow from an inverse square law of attraction. A fully rigorous and complete treatment of the problem of the motion of two bodies under Newtonian gravitation was given by Leonhard Euler in 1744. The extension to more than two bodies has proved considerably less tractable. While excellent approximate methods exist (and are widely used in astronomy and space exploration), general theoretical insights seem hard to come by. The problem is deep and difficult, the phenomena are complex. Just how complex is shown by J. Gerver in the Journal of Differential Equations 52, 76 (1984).

Gerver gives a heuristic but convincing argument for the existence of configurations of five point masses which, under their mutual Newtonian gravitational attraction, fling themselves out to infinity in a finite time. This relates to an important theoretical issue: the possible occurence of singularities, that is events beyond which the motion cannot be continued within the mathematical model. In 1897, Paul Painlevé proved that for three bodies, every singularity must be a collision. Collision singularities are fairly well behaved: in fact if only two bodies collide at a given instant, then the motion can be 'desingularized' by assuming elastic collisions. But Painlevé also suggested that with more than three bodies there might exist much nastier types of singularity such as bodies escaping to infinity in a finite time, or oscillating ever more violently.

In 1970, H.J.Sperling proved that in order to have a singularity without collisions, at least one body must escape to infinity; and seven years later D. G. Saari showed that one of the escapees would have to oscillate wildly. He also showed that, in the case of four bodies, they would have to approach a straight line arrangement as time increased. In 1974, J. Mather and $\mathbf{R}$. McGehee, studying an idealization, showed that escape to infinity in finite time 